\title{
Precision Calorimetry for Power Loss Measurement of a Very Low Power Maximum Power Point Tracker
}

\author{
Peter Wolfs and Quan Li \\ Central Queensland University \\ Rockhampton Mail Center, QLD 4702, Australia
}

\begin{abstract}
Efficiency is an important performance measure for dc-de converters. Its measurement can be extremely difficult when the dc-dc converter is highly efficient and the power loss is extremely low. The input and the output waveforms of a high frequency dc-dc converter may have high frequency components superimposed on the de components. This further complicates the measurement process as some digital instruments tend to misbehave. Calorimetry method has been previously proposed as an alternative and indirect power loss measurement method. This paper proposes an improved calorimetry method which is capable of resolving the power loss below $2 \mathrm{~mW}$. The method is demonstrated for a single-cell maximum power point tracker with a power rating of $650 \mathrm{~mW}$. The experimental results confirm that the device under test presents a power loss of $24 \mathrm{~mW} \pm 1.3 \mathrm{~mW}$ and the converter achieves an efficiency of $96.2 \% \pm 0.2 \%$.
\end{abstract}

\section{INTRODUCTION}

One important task in the evaluation of the performances of the power electronic converters is to establish the overall power loss or power conversion efficiency. However, the efficiency measurement of high performance power electronic converters becomes increasingly difficult as the converter efficiency rises. The error in the power loss measurement is the sum of the errors in the input and the output power measurements. As the efficiency rises, the power losses fall and the percentage error in the power loss measurement increases. In addition, the converter input and output waveforms may contain high frequency components, which may lead to power transfer and further complicate the power loss measurement process. Some digital instruments may misbehave under these circumstances.

In a study of the Maximum Power Point Tracking (MPPT) hardware design for vehicle solar array applications, the power loss measurement task became onerous. In this study, a maximum power point tracker targeting at the single solar cell level has been proposed to obtain excellent power tracking performances [1]-[3]. The small and cost effective maximum power point tracker is capable of being integrated with a single high performance GaAs triple junction cell [4]. Since the vehicle mounted cells can travel through the alternating light and shaded sections very quickly, significant efforts have been made to secure a fast power tracking response to the rapidly changing insolation conditions, preferably within a few milliseconds. The design of the maximum power point tracker also demands a high power conversion efficiency or a low power loss since the converter power rating falls in the milliwatt range. An estimation of the total power loss is around
$20 \mathrm{~mW}$ for the $650-\mathrm{mW}$ converter and this needs to be validated by experimental measurement. The extremely low power loss creates a further challenge in the power loss measurement. A highly accurate measurement method must be employed to complete the task.

In the design of the maximum power tracker, an inductorless buck converter topology was selected in order to optimize the size of the individual device. The circuit diagram is shown in Fig. 1. However, this design further complicates the power loss measurement as significant switching frequency components will be present in the output voltage and current waveforms and a significant amount of power may be transferred to the load at frequencies other than dc.

Calorimetry has been widely accepted as an alternative and indirect power loss measurement method for converters and rotating machines [5]-[10]. This paper proposes an improved calorimetry method which is capable of resolving power losses below $2 \mathrm{~mW}$. The experimental setup is studied in detail and the important design considerations are discussed. Finally, the power loss measurement data for a maximum power point tracker with $650-\mathrm{mW}$ power rating are provided. The experimental results confirm that the device under test has achieved a power loss of $24 \mathrm{~mW}$ and the measurement accuracy is $\pm 1.3 \mathrm{~mW}$. Under an input power of $652 \mathrm{~mW}$, this is equivalent to a power conversion efficiency of $96.2 \% \pm 0.2 \%$.

\section{Fundatmental CALORIMETRY MethoD}

Fundamental calorimetry method has been previously proposed as an accurate measurement method for the power loss in efficient dc-dc converters, as the determination of the power loss is extremely difficult simply from the converter input and output measurements. When measuring the power loss of high frequency dc-dc converters, some digital

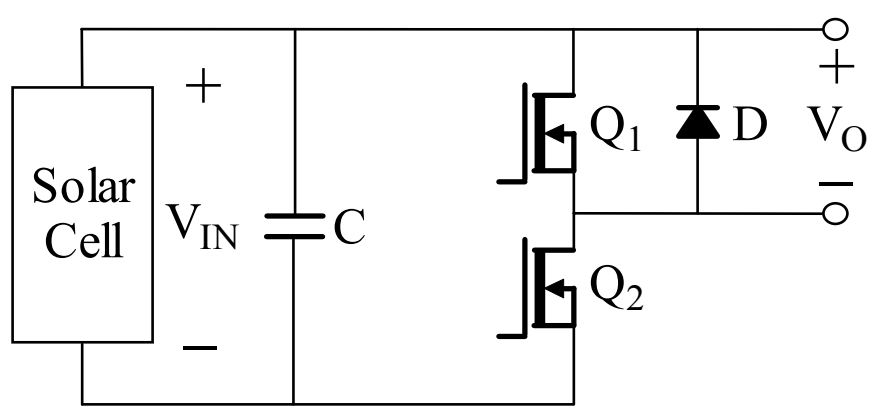

Fig. 1. Inductorless Buck Converter 
instruments will misbehave due to the high frequency components in the converter input and/or output and accurate results cannot be obtained. Calorimetry method provides an indirect power loss measurement method and it can achieve a significantly higher measurement accuracy. The measurement must be conducted in a closed insulated container. Other devices installed inside the container include:

- A fan, which is used to stir the air inside so that the thermal equilibrium can be reached faster and no hot spot exists,

- Several temperature sensors, such as National Semiconductor LM35 [11], which are used to measure the temperatures of different spots inside the container,

- The device under test, which is the dc-dc converter or the rotating machine, and

- A power resistor with the power rating of at least the estimated total loss of the device under test, which is used in the system calibration process.

At least one temperature sensor has to be installed outside to measure the ambient temperature. In order to obtain accurate results, the container must be sealed before the experiment is carried out and the enclosure must remain undisturbed during the entire experiment. The experiment proceeds in two stages: the system calibration stage and the power loss measurement stage.

In the system calibration stage, different input voltages are applied to the power resistor to obtain a range of power consumption values. It is expected that the actual power loss of the device under test will fall within this range. The temperature rise is obtained after thermal equilibrium is reached and by calculating the difference between the average of the stabilized temperatures shown by the inside sensors and the temperature shown by the outside sensor. Data of a series of the temperature rises against the corresponding power inputs are recorded and a linear graph, a straight line for the two variables, can be produced.

The next stage is the actual power loss measurement of the device under test. In this stage, the voltage source is disconnected from the power resistor and applied at the device under test. When the whole system reaches its thermal equilibrium, the temperature rise is recorded. By comparing this temperature rise with the calibration graph, the total power loss of the device can be obtained.

One significant advantage of the calorimetry method is that it does not demand an extremely high insulation resistance for the enclosure. The accuracy of the measurement will not be affected if the enclosure remains undisturbed during the entire experiment. The small amount of the heat transfer will be taken into account when the calibration graph is generated. However, the fluctuation of the ambient temperature does have an adverse effect on the accuracy of the power loss measurement.

\section{IMPROVED CALORIMETRY METHOD}

Although the fundamental calorimetry method is able to provide a much more accurate power loss measurement for dcdc converters than the conventional voltage and current measurement, it is impossible to measure the extremely low power loss in the $650-\mathrm{mW}$ maximum power point tracker due to the following reasons:

- The variation of the ambient temperature may be significant compared with the temperature rise inside the insulated container.

- The power loss in the fan will be significantly higher than the estimated power loss in the MPPT hardware.

- The temperature sensor LM35 only has a measurement accuracy of $0.5^{\circ} \mathrm{C}$ and has a self temperature increase of $0.08{ }^{\circ} \mathrm{C}$. These may be comparable to the temperature rise caused by the estimated power loss of $20 \mathrm{~mW}$ in the MPPT hardware.

In the improved calorimetry method, a much more stable environment is required as the accuracy of the calorimetry method is extremely sensitive to the temperature differential between the system under test and the ambient. The improved calorimetry method proposed in this paper conducts the test inside a thermos flask. In this case, the thermos flask does not have to be a perfect insulator as long as the surrounding temperature is constant. In order to maintain the outside temperature constant, the thermos flask is placed inside a polystyrene container with a significant internal thermal mass. In this case, two litres of water, in a plastic bottle, is used. The polystyrene container is additionally protected from temperature fluctuation by placing it inside an environmental chamber. In this experiment, the Contherm 5200RHS environmental chamber is used and its temperature controller has an accuracy of $\pm 0.3{ }^{\circ} \mathrm{C}$ [12]. The environmental chamber is also fitted with a $50-\mathrm{mm}$ cable port with good insulators as blocks, which allows the electrical cables from the internal circuits to be connected to the external power supplies, inductive resistive load and data acquisition system. The temperature of the environmental chamber is set to $27^{\circ} \mathrm{C}$ in the experiment, which is slightly higher than the room temperature, to avoid the delay of the temperature controller's response when it changes between the heating and the cooling cycles.

In the proposed method, the stirring fan is eliminated and 50 millilitres of transformer oil is used instead. In the thermos flask, the device under test and the temperature sensors are submerged in the transformer oil, which provides good thermal coupling without the stirring power.

One significant modification in the precision calorimetry method is the design of the temperature sensing process. A precise differential temperature measurement circuit made of a Wheatstone bridge consisting of four Pt100 platinum sensing resistors and a chopper-stabilized operational amplifier, MAX430 is employed. Fig. 2 shows the circuit diagram. At the single device quantity, the amplifier MAX430 and the sensing resistor Pt100 respectively cost around \$20 and \$10 AU [13]. The photo of the temperature measurement circuit is shown in Fig. 3 and this is located outside the chamber. The four sensing 
resistors in Fig. 2 are located inside the chamber and remotely connected to the circuit board.

In Fig. $2, \mathrm{R}_{1}$ to $\mathrm{R}_{4}$ are the platinum sensing resistors and these are $100 \Omega$ each at $0{ }^{\circ} \mathrm{C}$ [14]. Each resistor has a temperature coefficient of $+0.385 \Omega /{ }^{\circ} \mathrm{C}$. Resistors $\mathrm{R}_{1}$ and $\mathrm{R}_{2}$ are located outside the thermos flask, while resistors $R_{3}$ and $R_{4}$ are located inside the thermos flask and submerged in the transformer oil. This arrangement makes the bridge output only sensitive to the temperature difference between the contents of the thermos flask and the surrounding environment. The output voltage of the bridge circuit has a linear relationship with the temperature difference. The voltage and the differential temperature ratio is $0.193 \mathrm{mV} /{ }^{\circ} \mathrm{C}$ at $0{ }^{\circ} \mathrm{C}$. Since the two of the four resistors in the Wheatstone bridge are placed inside the thermos flask, the power dissipation must be designed to be significantly smaller than the estimated power loss of the device under test. In the arrangement shown in Fig. 2, the bridge circuit is connected in series with larger resistors so that it can be driven by the $5-\mathrm{V}$ power supply. At $0{ }^{\circ} \mathrm{C}$, a current of $1 \mathrm{~mA}$ flows through the bridge circuit and results in a total power dissipation of $50 \mu \mathrm{W}$ in $\mathrm{R}_{3}$ and $\mathrm{R}_{4}$. The power dissipation will be slightly increased at higher temperatures in the test due to their positive temperature coefficient. The effect of the any power dissipation in $\mathrm{R}_{1}$ and $\mathrm{R}_{2}$ will be cancelled by the calibration process.

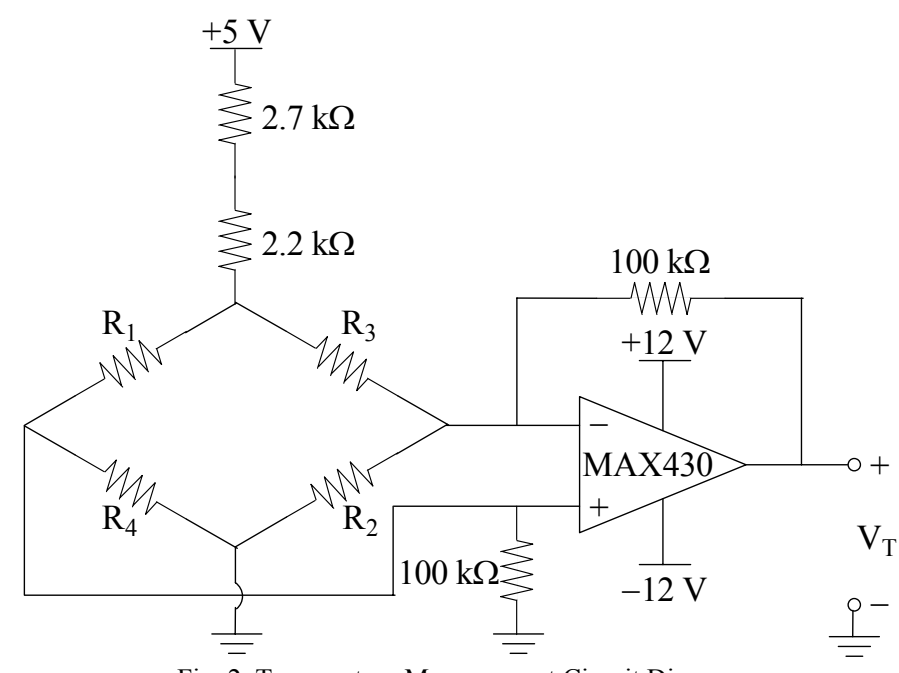

Fig. 2. Temperature Measurement Circuit Diagram

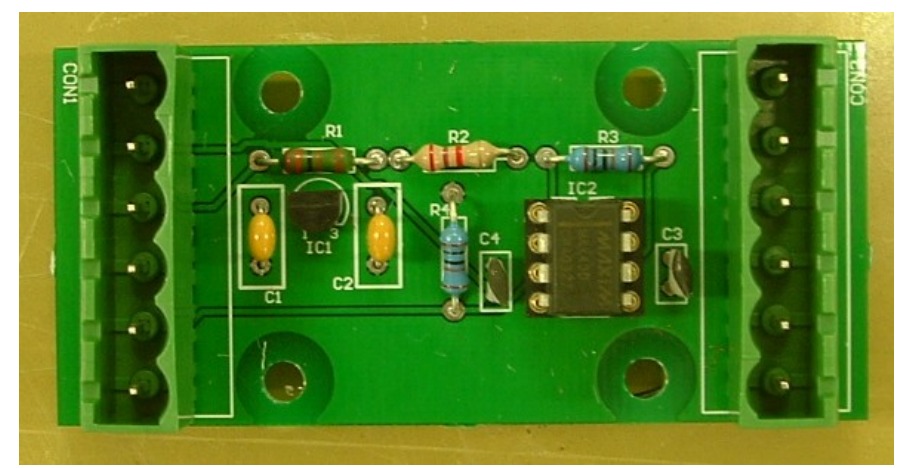

Fig. 3. Photo of the Temperature Measurement Circuit
The MAX430 is a precision amplifier and its cost is significantly higher than the standard operational amplifiers. However, the normal operational amplifier has a high temperature coefficient of input voltage offset. For example, National Semiconductor TL081 has an average temperature coefficient of input voltage offset of $10 \mu \mathrm{V} /{ }^{\circ} \mathrm{C}$ [15]. The temperature variation outside the environmental chamber will cause significant input voltage variation compared with the voltage and the differential temperature ratio of $0.193 \mathrm{mV} /{ }^{\circ} \mathrm{C}$ of the Wheatstone bridge. The MAX430 has only a maximum $50-\mathrm{nV} /{ }^{\circ} \mathrm{C}$ average temperature coefficient of input voltage offset [16]. The maximum input bias current is $100 \mathrm{pA}$ at $25^{\circ} \mathrm{C}$ and this has a negligible effect. As the impedance at the amplifier input terminals is around $100 \Omega$, the voltage due to the bias current is about $10 \mathrm{nV}$. The amplifier has a differential gain of 1000 or a voltage temperature sensitivity of $193 \mathrm{mV} /{ }^{\circ} \mathrm{C}$.

In the improved calorimetry method, the resistive load used to obtain the calibration curve must have a low tolerance in order to obtain a highly accurate power loss measurement. In this experiment, a $1-\mathrm{k} \Omega$ resistor with $1 \%$ tolerance is employed. As an example, the application of $5 \mathrm{~V}$ across the resistor results in a well defined $25-\mathrm{mW}$ power dissipation. An observation of the steady state temperature rise provides a single calibration point.

It is worth mentioning that care must be taken when selecting the electrical cables to connect the input and the output terminals of the MPPT device to the external power supply and load. The power dissipation from the cable can be significant compared with the estimated power loss of the MPPT device itself. For example, under an input current of $300 \mathrm{~mA}$ and a switching duty ratio of $50 \%, 10-\mathrm{m} \Omega$ input cable resistance and $10-\mathrm{m} \Omega$ output cable resistance inside the thermos flask lead to a power dissipation of $4.5 \mathrm{~mW}$. Therefore, thicker cables made of high-conductivity conductors are preferred. The conductors also impact on the thermal conductivity but this is captured by the calibration process.

\section{EXPERIMENTAL RESULTS}

Fig. 4 illustrates the system configuration of the improved calorimetry method. The electrical cables coming out of the environmental chamber connect to the power supply, the load and the data acquisition card. The photos of the laboratory implementations are shown in Figs. 5 and 6. In the improved calorimetry method, the data of the differential voltage output from the temperature sensing circuit shown in Fig. 2 are recorded by the National Instrument LabVIEW system to allow unattended operation [17]. One voltage reading is automatically logged every 15 minutes and a 24 -hour period is used to measure the differential voltage of each input power value. Over the 24 hours, the first 21 hours allow the system to reach the thermal equilibrium and 12 data recorded during the last three hours will be used to establish the calibration curve or the power loss of the MPPT hardware.

Table I lists the measurement data of the initial offset, the ten calibration points and the MPPT converter. Table II lists the data used in the calibration curve. The input power is 
calculated from the input voltage on the $1-\mathrm{k} \Omega$ resistor and the average differential voltage is obtained by subtracting the average offset voltage from the average measured voltage values. Fig. 7 shows the calibration curve in the calorimetry measurement, where the horizontal axis $\mathrm{P}_{\text {loss }}$ is the power dissipation in the system and the vertical axis $V_{T}$ is the differential voltage. Error bars representing the positive and the negative errors at the individual calibration points are also shown in Fig. 7. A maximum differential voltage measurement variation of $16 \mathrm{mV}$ has been observed and this is equivalent to a measurement accuracy is $\pm 1.3 \mathrm{~mW}$. This also corresponds to the accuracy of the thermos flask differential temperature of $\pm 0.08^{\circ} \mathrm{C}$, which is significantly better than that of the LM35.

Consider the offset value, the average differential voltage when running the MPPT converter is $318 \mathrm{mV}$. Compared with the calibration curve in Fig. 7, the results confirm that the MPPT converter has a power loss of $24 \mathrm{~mW} \pm 1.3 \mathrm{~mW}$.

In the process of measuring the power loss of the MPPT converter, the input current is established from the voltage across two series connected $1-\Omega$ resistors with $1 \%$ tolerance. The converter input voltage is $2.281 \mathrm{~V}$ and the input current is $0.286 \mathrm{~A}$. Under this input power of $652 \mathrm{~mW}$, it can be validated that the converter has achieved an efficiency of $96.2 \% \pm 0.2 \%$.

\section{CONCLUSIONS}

Fundamental calorimetry method provides an alternative approach in measuring the power loss in the efficient power electronic converters and rotating machines. This paper studies an improved calorimetry method with a much better precision. The experimental setup of the improved method is discussed in detail. Finally, the method is demonstrated for a $650-\mathrm{mW}$ MPPT hardware and establishes a power loss of $24 \mathrm{~mW} \pm 1.3$ $\mathrm{mW}$. To obtain a similar level of accuracy by direct measurements of the input and the output powers, each would need to be determined with an error of $\pm 0.65 \mathrm{~mW}$ or $\pm 0.1 \%$ of the rated power of the MPPT converter. This level of experimental accuracy allows meaningful comparisons of the power loss changes due to design variations in a range of the MPPT converters. The proposed method can be also applied to the power loss measurement of other efficient low-power converters and obtain accurate results.

\section{ACKNOLEDGEMENT}

This work was supported by the Queensland Department of Public Works and Housing and the Queensland Department of Transport.

\section{REFERENCES}

[1] P. Wolfs and L. Tang, "A single cell maximum power point tracking converter without a current sensor for high performance vehicle solar arrays," in Proc. IEEE PESC, 2005, pp. 165-171.

[2] P. Wolfs and Q. Li, "A Current-Sensor-Free Incremental Conductance Single Cell MPPT for High Performance Vehicle Solar Arrays," in Proc. IEEE PESC, 2006, pp. 117-123.

[3] P. Wolfs and Q. Li, "Performance analysis and hardware implementation of a current-sensor-free single cell MPPT for high performance vehicle solar arrays," in Proc. IEEE PESC, 2007, pp. 132137.

[4] Spectrolab Inc. (2002, May). 25.1\% GaInP2/GaAs/Ge triple junction solar cells. [Online]. Available: http://www.spectrolab.com/DataSheets/ TJCell/tj.pdf

[5] K. J. Bradley, A. Ferrah, R. Magill, J. C. Clare, P. Wheeler and P. Sewell, "Precision calorimetry for loss evaluation," in Proc. IEE Half Day Colloquium on Testing of Electrical Machines, 1999, pp. 3/1-3/3.

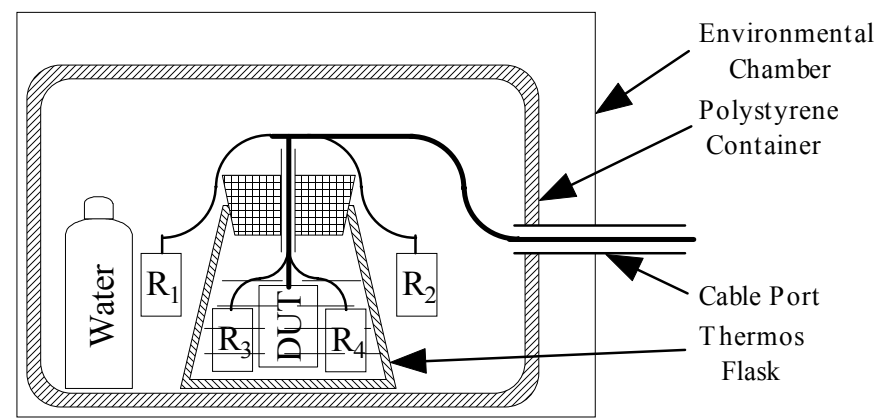

Fig. 4. Measurement System Configuration

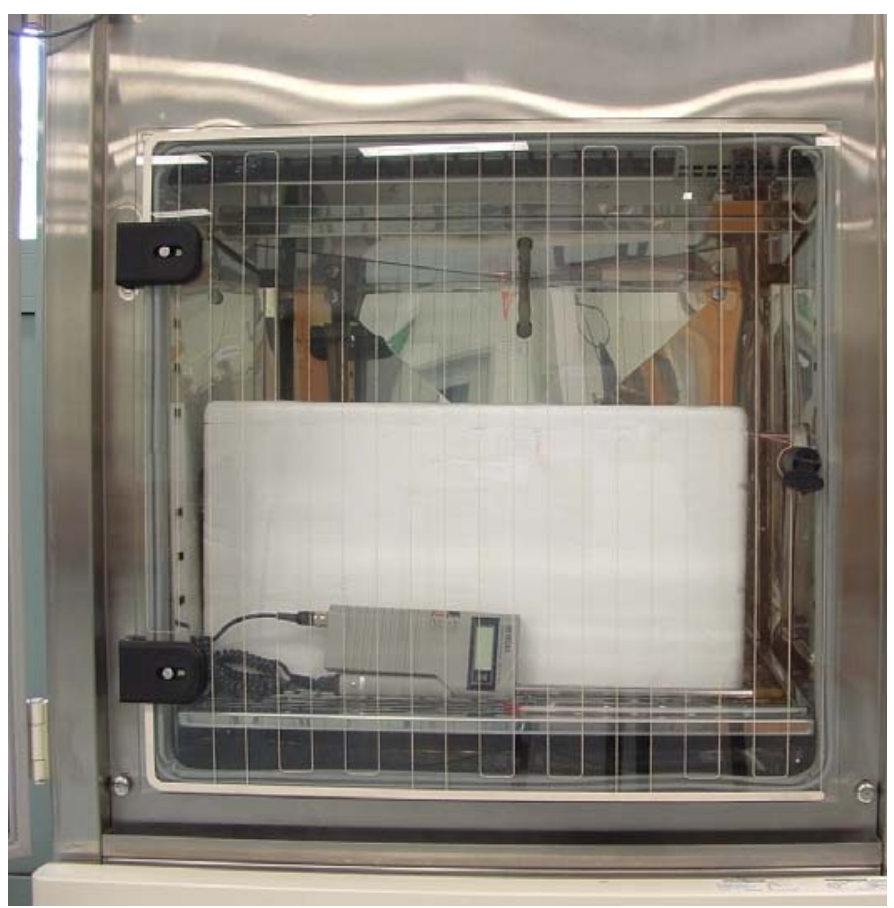

Fig. 5. Environmental Chamber

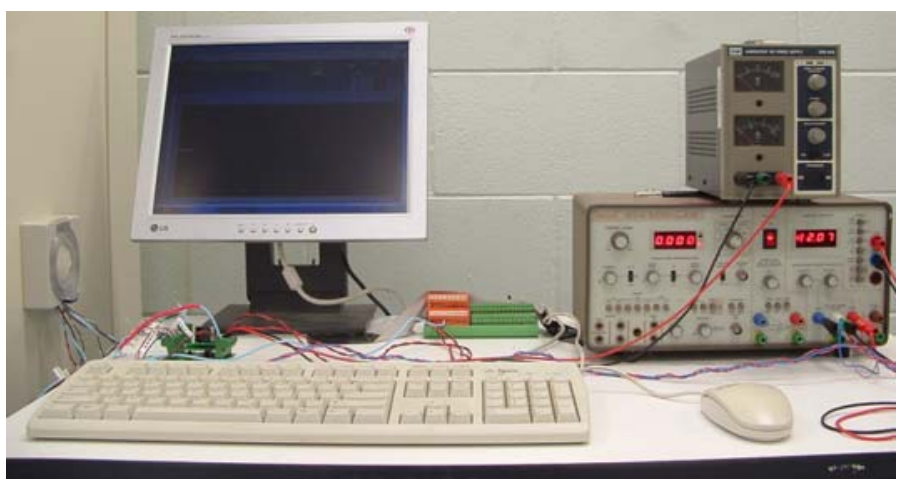

Fig.6. Test Bench 
TABLE I

DATA RECORDED BY LABVIEW

\begin{tabular}{|c|c|c|c|c|c|c|c|c|c|c|c|c|c|}
\hline & Measurement & Offset & 1 & 2 & 3 & 4 & 5 & 6 & 7 & 8 & 9 & 10 & Converter \\
\hline \multicolumn{2}{|c|}{ Input Voltage (V) } & 0.000 & 2.241 & 3.169 & 3.873 & 4.471 & 5.001 & 5.477 & 5.918 & 6.328 & 6.708 & 7.080 & 2.281 \\
\hline \multirow{12}{*}{ 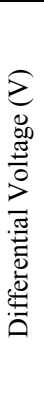 } & Recording 1 & 0.149 & 0.228 & 0.288 & 0.353 & 0.411 & 0.47 & 0.544 & 0.605 & 0.669 & 0.736 & 0.793 & 0.472 \\
\hline & Recording 2 & 0.149 & 0.226 & 0.287 & 0.352 & 0.411 & 0.466 & 0.541 & 0.608 & 0.667 & 0.733 & 0.787 & 0.475 \\
\hline & Recording 3 & 0.149 & 0.225 & 0.287 & 0.351 & 0.411 & 0.469 & 0.535 & 0.606 & 0.666 & 0.731 & 0.786 & 0.477 \\
\hline & Recording 4 & 0.150 & 0.224 & 0.287 & 0.350 & 0.411 & 0.473 & 0.528 & 0.603 & 0.665 & 0.728 & 0.787 & 0.474 \\
\hline & Recording 5 & 0.150 & 0.223 & 0.286 & 0.348 & 0.412 & 0.477 & 0.528 & 0.600 & 0.663 & 0.726 & 0.790 & 0.469 \\
\hline & Recording 6 & 0.150 & 0.222 & 0.286 & 0.348 & 0.413 & 0.478 & 0.529 & 0.594 & 0.662 & 0.725 & 0.786 & 0.466 \\
\hline & Recording 7 & 0.150 & 0.222 & 0.285 & 0.348 & 0.413 & 0.475 & 0.533 & 0.593 & 0.660 & 0.724 & 0.782 & 0.461 \\
\hline & Recording 8 & 0.151 & 0.221 & 0.285 & 0.348 & 0.413 & 0.472 & 0.537 & 0.594 & 0.659 & 0.724 & 0.791 & 0.461 \\
\hline & Recording 9 & 0.152 & 0.221 & 0.285 & 0.348 & 0.413 & 0.469 & 0.539 & 0.598 & 0.660 & 0.722 & 0.787 & 0.464 \\
\hline & Recording 10 & 0.152 & 0.221 & 0.285 & 0.348 & 0.415 & 0.466 & 0.535 & 0.601 & 0.660 & 0.722 & 0.784 & 0.469 \\
\hline & Recording 11 & 0.152 & 0.221 & 0.286 & 0.348 & 0.415 & 0.469 & 0.531 & 0.601 & 0.660 & 0.721 & 0.783 & 0.469 \\
\hline & Recording 12 & 0.152 & 0.220 & 0.286 & 0.347 & 0.416 & 0.473 & 0.528 & 0.598 & 0.659 & 0.72 & 0.782 & 0.469 \\
\hline
\end{tabular}

TABLE II

Calibration CuRve Data

\begin{tabular}{|c|c|c|c|c|c|c|c|c|c|c|c|}
\hline \multicolumn{2}{|c|}{ Calibration Point } & 1 & 2 & 3 & 4 & 5 & 6 & 7 & 8 & 9 & 10 \\
\hline \multicolumn{2}{|c|}{ Input Power (mW) } & 5.02 & 10.04 & 15.00 & 19.99 & 25.01 & 30.00 & 35.02 & 40.04 & 45.00 & 50.13 \\
\hline \multirow{4}{*}{$\begin{array}{c}\text { Differential } \\
\text { Voltage } \\
(\mathrm{mV})\end{array}$} & Average Value & 72 & 135 & 198 & 262 & 320 & 383 & 449 & 512 & 575 & 636 \\
\hline & Positive Error & 5 & 2 & 4 & 3 & 7 & 10 & 8 & 7 & 10 & 6 \\
\hline & Negative Error & 3 & 1 & 2 & 2 & 5 & 6 & 7 & 3 & 6 & 5 \\
\hline & Total Variation & 8 & 3 & 6 & 5 & 12 & 16 & 15 & 10 & 16 & 11 \\
\hline
\end{tabular}

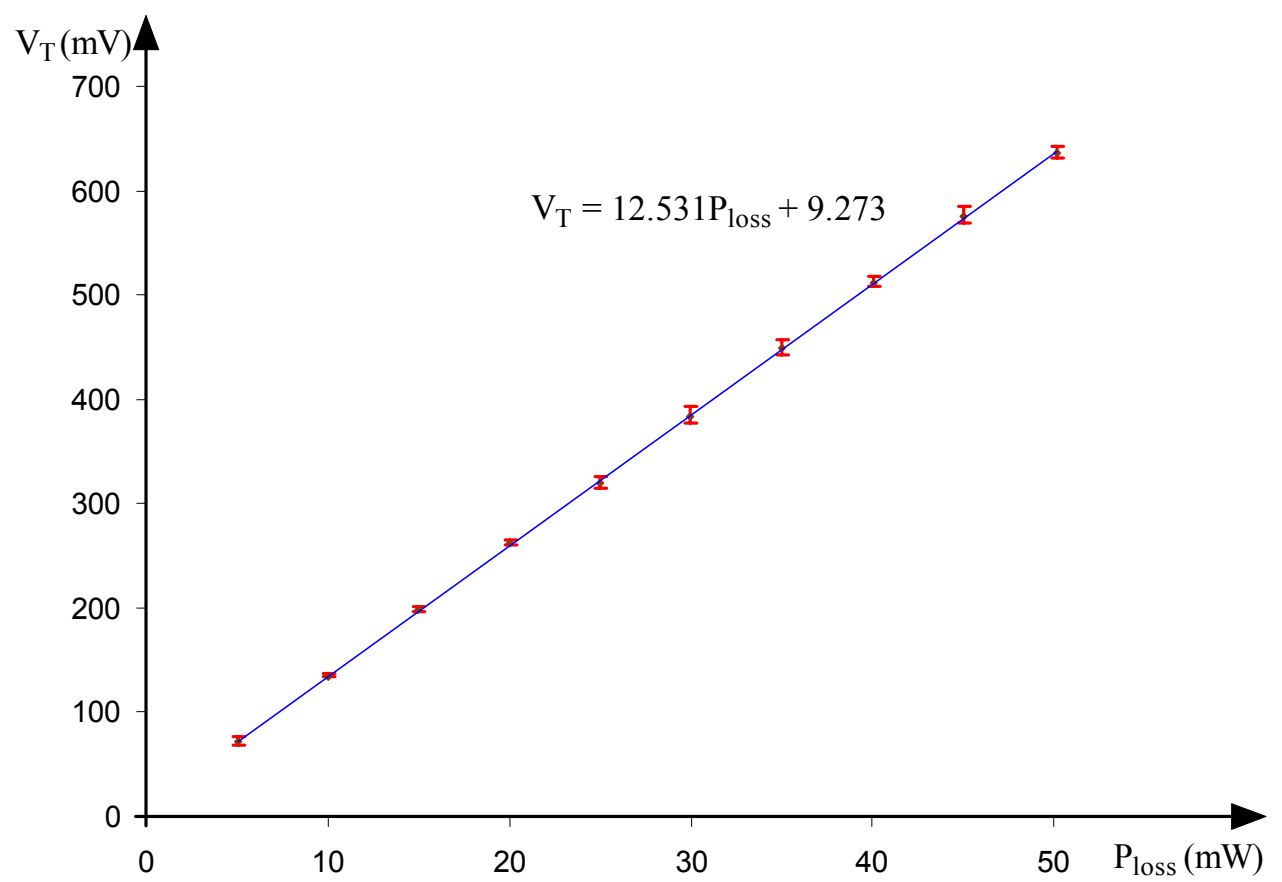

Fig. 7. Calorimetry Calibration Curve

[6] D. Patterson, "Simple calorimetry for accurate loss measurement," IEEE Power Electronic Society Newsletter, pp. 5-7, Oct. 2000.

[7] Q. Li, "Development of high frequency power conversion technologies for grid interactive PV systems," Master of Engineering Dissertation, Central Queensland University, Australia, 2002.

[8] P. D. Malliband, N. P. van der Duijn Schouten and R. A. McMahon, "Precision calorimetry for the accurate measurement of inverter losses," in Proc. IEEE PEDS, 2003, pp. 321-326.

[9] S. D. J. Weier, R. A. McMahon, and P. D. Malliband, "Calorimetry for power electronics," in Proc. Universities Power Engineering Conf., 2006, pp.608-612.

[10] National Semiconductor. (2000, Nov.). LM35 precision centigrade temperature sensors. [Online]. Available: http://cache.national.com/ ds/LM/LM35.pdf
[11] Contherm Scientific. (2005, Mar.). Series 5000 environmental chambers. [Online]. Available: http://www.contherm.co.nz/Brochures/ 5000RHS.pdf

[12] RS Components. [Online]. Available: http://www.rsaustralia.com/

[13] Labfacility LTD. Pt100 datasheet. [Online]. Available: http://www.temperature-sensors 1. com/pdf/sensors-PRTs.pdf

[14] National Semiconductor. (1995, Dec.). TL081 wide bandwidth JFET input operational amplifier. [Online]. Available: http://cache.national.com/ds/TL/TL081.pdf

[15] Maxium Integrated Products. (1998, Aug.). Max430/432 datasheet. [Online]. Available: http://datasheets.maxim-ic.com/en/ds/MAX430MAX432.pdf

[16] National Instrument. LabVIEW. [Online]. Available: http://www.ni.com/ labview 